Ophthalmologe 2022 $\cdot 119: 752-756$ https://doi.org/10.1007/s00347-021-01534-x Eingegangen: 8. Oktober 2021 Überarbeitet: 26. Oktober 2021 Angenommen: 28. Oktober 2021 Online publiziert: 29. November 2021 (c) Der/die Autor(en) 2021

\section{Akute einseitige Protrusio bulbi}

\author{
K. Borgardts · K. Spaniol · C. Trigaux · W. Dahma · M. Kaschner · M. Borrelli · G. Geerling \\ Universitätsklinikum Düsseldorf, Düsseldorf, Deutschland
}

Die Autoren M. Borrelli und G. Geerling haben die geteilte Senior-Autorenschaft.

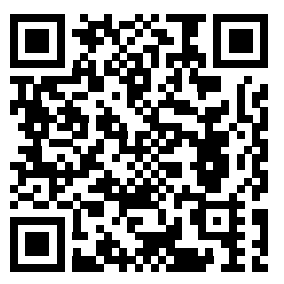

QR-Code scannen \& Beitrag online lesen

\section{Anamnese}

Eine 90-jährige Frau stellte sich mit plötzlichem Exophthalmus rechts seit dem Morgen vor (• Abb. 1). Zudem gab die Patientin ein geringes Schwellungsgefühl im Bereich der rechten Gesichtshälfte und drückende Schmerzen periokulär rechts an. Am Abend zuvor sei ihr beim Fernsehen eine leicht unscharfe Sicht rechts aufgefallen, Doppelbilder bestünden nicht. Ein Trauma sei nicht erinnerlich. Bis auf eine Kataraktoperation 2015 auf dem rechten Auge war die Augenanamnese unauffällig. Links war eine Amblyopie bekannt. Sie habe kein Fieber, keine Kopfschmerzen, keinen Infekt im Gesichtsbereich gehabt und sei Nichtraucherin. Bekannt waren hingegen ein Asthma bronchiale, eine Hypothyreose und eine Spondylitis ankylosans, die mit Beclometason, Formoterol (Inhalationsspray) und L-Thyroxin behandelt wurden.

\section{Befund bei Erstvorstellung}

In der klinisch-ophthalmologischen Untersuchung zeigte sich ein bestkorrigierter Visus von 0,63 rechts und 1/40 Metervisus links sowie ein Exophthalmus von $5 \mathrm{~mm}$ rechts, gemessen mit dem HertelExophthalmometer (rechts $18 \mathrm{~mm}$, links $13 \mathrm{~mm}$, Basis $117 \mathrm{~mm}$ ). Der vordere Augenabschnitt rechts zeigte eine Bindehautchemosis und einen Nachstar. Links waren Lider und Bindehaut reizarm, die Hornhaut war klar, glatt, spiegelnd mit Arcus senilis, die Vorderkammer mitteltief und reizfrei, die Iris altersgerecht, und es zeigte sich eine provekte Katarakt mit Vakuolen. Fundoskopisch bestanden keine Auffälligkeiten. Die Pupillen waren isokor mit einem relativen afferenten Pupillendefizit (RAPD) rechts ohne Rotentsättigung. Es zeigte sich kein Lagophthalmus, das Bell-Phänomen war positiv. Die Hornhautsensibilität war rechts in allen Quadranten intakt.

Nach Erhebung des orthoptischen Status drei Tage nach Symptombeginn bestand der Verdacht auf eine sekundäre Exotropie in der Ferne auf dem linken Auge, bei jedoch schlechter Fixationsaufnahme links. In der Nähe zeigte sich eine geringe Exotropie auf dem linken Auge mit strenger Rechtsführung ohne Wahrnehmung von Doppelbildern. Die Motilität war unauffällig. Der Bagolini-Test ergab für die Nähe eine Suppression des linken Auges, auch gemessen mit dem Hellrotglas. Es wurde eine geringe Anisokorie links größer als rechts bei beidseits guter Lichtreaktion beobachtet. Nach Kestenbaum ergab sich ein Tieferstand des rechten Auges von $4 \mathrm{~mm}$.

Die körperliche Untersuchung ergab bis auf eine leichte Gesichtsschwellung periorbital rechts keine Auffälligkeiten. Die Laborergebnisse vom Aufnahmetag waren bis auf leicht erhöhte Erythrozyten(5,31 Mio./ $\mu \mathrm{l})$, Hämoglobin- (15,7 g/dl) und Hämatokritwerte $(47,6 \%)$ unauffällig.

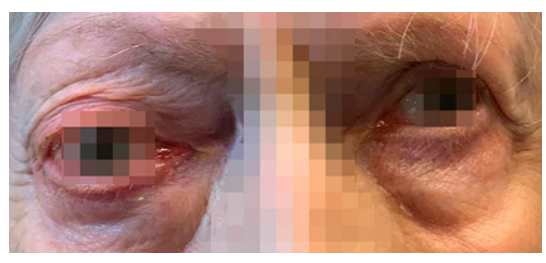

Abb. 1 \ Erstvorstellung, Porträtaufnahme. Am rechten Auge zeigt sich ein Exophthalmus sowie Tieferstand 

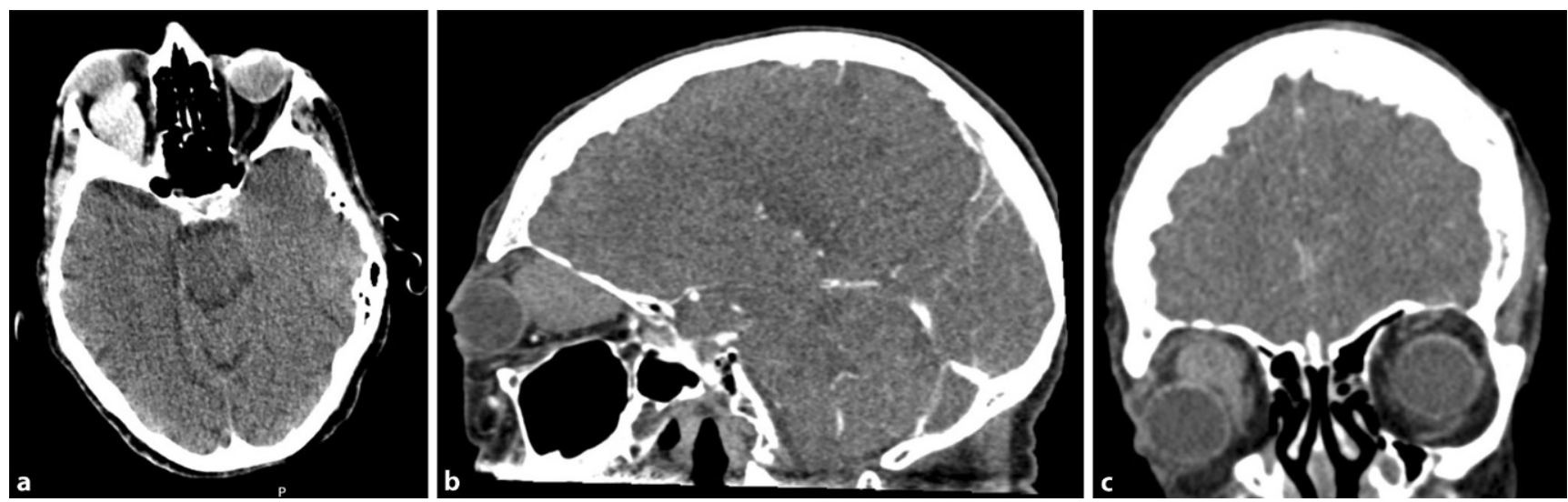

Abb. 2 ム CT-Aufnahmen des Schädels am Aufnahmetag. Es zeigt sich eine intrakonale Raumforderung rechts mit resultierender Protrusio bulbi in axialer (a) sowie sagittaler Ebene (b) und in der koronaren Schnittführung (c)

\section{Weitere Diagnostik}

In der noch am selben Tag durchgeführten Computertomographie(CT)-Aufnahme des Schädels und der Orbita zeigte sich rechts eine ausgedehnte, intrakonale Raumforderung von ca. $34 \times 22 \times 10 \mathrm{~mm}$ ohne Dynamik nach Kontrastmittelgabe mit resultierender Protrusio bulbi sowie Verdrängung des $M$. rectus superior in
Verlängerung eines am ehesten venösen Gefäßes ( Abb. 2a-c), vermutlich der V. ophthalmica.

\section{Wie lautet Ihre Diagnose?}

Diagnose. Rechtes Auge: Exophthalmus und Hypoglobus bei Thrombose der V. ophthalmica.

Therapie. Nach interdisziplinärer Rücksprache erfolgte eine aktivierte partielle Thromboplastinzeit(aPTT)-adaptierte Vollantikoagulation mit unfraktioniertem Heparin. Fünf Tage später wurde eine Therapie mit Rivaroxaban $15 \mathrm{mg} / \mathrm{Tag}$ eingeleitet und das unfraktionierte Heparin abgesetzt.

Verlauf. Die Patientin wurde zehn Tage später in die eigene Häuslichkeit entlassen, wobei der Befund bis auf einen leichten Rückgang der Protrusio bulbi mit einem Exophthalmus von $4 \mathrm{~mm}$ rechts dem Aufnahmebefund entsprach. Zum Entlasszeitpunkt zeigte sich noch ein angedeutetes RAPD rechts.

Ein Monat nach Symptombeginn zeigte sich bei deutlich besserem Befund und fehlender Beschwerdesymptomatik unter der Therapie mit Rivaroxaban ein Visus von rechts 0,7 und links 0,05 mit eigener Korrektur. Vorderer und hinterer Augenabschnitt waren beidseits unauffällig. Es bestand kein Exophthalmus mehr (• Abb. 3).

\section{Diskussion}

Da die venösen Sinus der Dura und die Hirnvenen keine Venenklappen aufweisen, bestimmen hauptsächlich Druckgradienten den Blutfluss [1]. Das venöse Blut kann daher im Kopf und in der Orbitaregion infolge einer venösen Obstruktion stagnieren [1]. Das spontane ein- oder beidseitige Auftreten einer Thrombose der V. ophthalmica superior ist ohne Assoziation mit einer Sinus-cavernosus-Thrombose selten [1-3].

Eine venöse Erkrankung der Orbitakann sich als arteriovenöse Malformation oder Fistel, als Thrombose des Sinus cavernosus oder der V. ophthalmica superior oder als orbitale Varix mit oder ohne Thrombose darstellen [1]. Eine Assoziation mit neoplastischen Prozessen ist beschrieben [1].

Die primäre Differenzialdiagnose war in diesem Fall eine orbitale Varix. Sie tritt typischerweise im mittleren Erwachsenenalter auf, kann sich jedoch auch kongenital manifestieren $[4,5]$.

Eine Varix ist die häufigste orbitale vaskuläre Malformation und auf bildgebenden Aufnahmen (Magnetresonanztomographie [MRT], CT) gut in Bauchlage zu sehen, da das Volumen der Varix in dieser
Position typischerweise zunimmt [6]. Ein positionsabhängiger, zum Teil schmerzhafter Exophthalmus, bei dem das Auge des Patienten sich nach außen verlagert, wenn er/sie sich nach vorne lehnt, ist somit ein kennzeichnendes Merkmal [6]. Eine venöse Varix zeigt sich als dilatierte venöse Struktur, die in Verbindung mit dem umliegenden venösen System steht, was in diesem Fall nicht vorlag. Auch eine Erhöhung des venösen Drucks in der Orbita im Rahmen eines Valsalva-Manövers kann den bildmorphologischen Nachweis einer orbitalen Varix unterstützen [7].

Krankheitsbild/Symptome. Patienten klagen typischerweise über Schmerzen und Schwellung im Orbita- oder Gesichtsbereich, Doppelbilder sowie Visusminderung $[1,3]$.

Häufig treten die Symptome beim Aufwachen auf, was darauf zurückzuführen sein könnte, dass eine Rückenlage zur venösen Stase beiträgt [2].

Okuläres Bild. Orbitale Stauungszeichen können sich unter anderem in Form einer Proptosis, Ptosis, Chemosis, Ophthalmoplegie, extraokularen Bewegungseinschränkung, episkleralen venösen Dilata- 


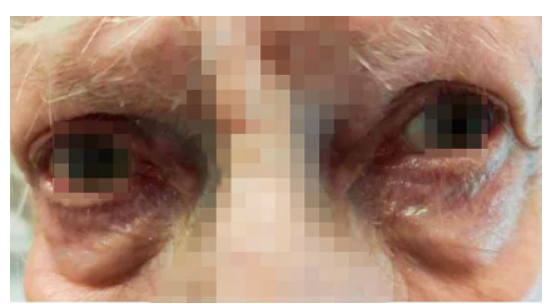

Abb. 3 ॥ Porträtaufnahme vier Monate nach Symptombeginn. Es lässt sich kein Exophthalmus mehr feststellen

tion (mit ggf. erhöhtem Augendruck durch erhöhte episklerale Druckverhältnisse), pathologischer Pupillenreaktion bei Affektion des N. opticus und Visusverlust zeigen [1-3].

Ätiologie. Die genaue Pathogenese einer spontanen Thrombose der V. ophthalmica superior ist bislang unklar und am ehesten multifaktoriell $[2,3]$. Es werden Thrombosen der V. ophthalmica superior septischer und aseptischer Genese unterschieden [8]. Infektionen mit Thrombophlebitis durch aerobe oder anaerobe Organismen sind die häufigste Ursache für eine Thrombose der V. ophthalmica superior $[3,8]$. Zu den aseptischen Ursachen zählt eine Hyperkoagulabilität, beispielsweise hervorgerufen durch eine Thrombozytose, Sichelzellenanämie, hereditäre hämorrhagische Teleangiektasie, Antiphospholipidsyndrom, Einnahme hormoneller Kontrazeptiva und Schwangerschaft [8]. Thrombosen der V. ophthalmica superior wurden zudem im Zusammenhang mit systemischem Lupus erythematodes, rheumatoider Arthritis, SjögrenSyndrom, Sinusitis oder einer Sinusoperation beobachtet [8].

Diagnostik. Bei Verdacht auf eine orbitale Gefäßerkrankung sollte nach Schmerzen und Diplopie, früheren Erkrankungen (z.B. Sinuserkrankung, Hyperkoagulabilität), Traumata und der Familiengeschichte in Bezug auf Gerinnungsstörungen gefragt werden [1]. Relevante systemische Begleiterkrankungen sollten ausgeschlossen werden [3]. Laborchemisch sollte eine Blutbildanalyse zum Ausschluss einer Leukozytose erfolgen [3]. Die Bildgebung des Kopfes (inklusive CT- und MRT-Aufnahme) spielt die entscheidende Rolle bei der Diagnosestellung, wobei das wichtigste bildgebende Merkmal die Dilatation der
V. ophthalmica superior mit inneren Läsionen darstellt [8].

Die wichtigste Differenzialdiagnose einer Dilatation der V. ophthalmica ist die Sinus-cavernosus-Fistel (SCF). In der CTBildgebung sind eine orbitale Kongestion mit Exophthalmus, eine retrobulbäre Verdichtung des Fettgewebes und Vergrößerung der Augenmuskeln zu sehen. Zu den vaskulären Veränderungen gehören eine Verdickung der V. ophthalmica superior sowie eine Vorwölbung und Asymmetrie des Sinus cavernosus mit Dichteanhebung der betroffenen Seite nach Kontrastmittelgabe (Dichte aufgrund der Arterialisierung vergleichbar mit der A. carotis interna). Der Ultraschall zeigt eine arterialisierte V. ophthalmica, und in der digitalen Subtraktionsangiographie (DSA) ist eine Shuntbildung zwischen $A$. carotis interna und S. cavernosus mit dilatierten Drainagevenen und Rückfluss in die V. ophthalmica nachweisbar.

\section{I) Diagnose: Exophthalmus und Hypoglobus bei Thrombose der V. ophthalmica}

Differenzialdiagnostisch kommen zahlreiche vaskuläre Pathologien der Orbita infrage. Hierbei wird zwischen vasoproliferativen Veränderungen und vaskulären Malformationen differenziert. Letztere werden in einfache (venöse, lymphatische, kapilläre, arteriovenöse Malformationen und arteriovenöse Fisteln), in kombinierte Malformationen, in solche von den großen Gefäßen ausgehend und Malformationen, die im Rahmen von Syndromen (z. B. Sturge-Weber-Syndrom) auftreten, unterteilt [9]. Die CT, die MRT und die Sonographie haben einen hohen Stellenwert in der Darstellung intraorbitaler vaskulärer Raumforderungen, da zum einen die Ausdehnung, der Bezug oder die Infiltration umgebender anatomischer Strukturen beurteilbar ist und zum anderen eine ätiologische Eingrenzung anhand der Morphologie, Signalveränderungen in der MRT und Kontrastmittelaufnahme möglich ist. Beispielsweise stellt sich die venöse Malformation (VM) als häufigster Typ der orbitalen vaskulären Malformationen in der MRT in den T1-gewichteten Sequenzen isointens zum Muskel mit homogener Kontrastmittelaufnahme und in den T2-gewichte- ten Sequenzen homogen hyperintens mit meist intrakonaler Lokalisation dar. Die CT kann durch Thrombosen bedingte Verkalkungen nachweisen [10]. Der Farbdoppler kann bei orbitalen vaskulären Malformationen eine Kommunikation mit dem arteriellen oder venösen System nachweisen, beispielsweise kann bei VM beim Valsalva-Manöver eine Distension und in der Duplexsonographie ein retrograder Fluss vorhanden sein. Dagegen sind lymphatische Malformationen (LM) nicht mit dem normalen Gefäßsystem der Orbita verbunden. In der Bildgebung zeigen sich bei der LM multikompartimentierte Raumforderungen, die oft sowohl den intra- als auch extrakonalen Raum betreffen und in der MRT oft ein buntes Bild aus hyper- und hypointensen Anteilen bedingt durch Blutungen unterschiedlichen Alters und nichtblutiger Flüssigkeit in den zystischen Komponenten aufweisen [11]. Die DSA hat aufgrund der guten diagnostischen Möglichkeiten der nichtinvasiven bildgebenden Verfahren in den meisten Fällen einen untergeordneten Stellenwert in der initialen Diagnostik orbitaler vaskulärer Pathologien. Dagegen besteht eine Indikation zur DSA, wenn arterialisierte Pathologien vorliegen. Hier hat die hohe räumliche und zeitliche Auflösung einen Vorteil gegenüber den nichtinvasiven Modalitäten und ist zur endovaskulären und operativen Behandlungsplanung wie z.B. bei der SCF oder einer arteriovenösen Malformation essenziell.

Therapiemöglichkeiten. Zur Behandlung und Prognose einer Thrombose der V. ophthalmica superior liegen hauptsächlich Fallberichte vor (s. - Tab. 1; [2, 8]). Grundsätzlich sollte sich die Behandlung nach der Ätiologie richten, was eine genaue Diagnosestellung unerlässlich macht $[1,3,8]$. Bei infektiöser Ursache sollte eine empirische Behandlung mit Breitspektrumantibiotika ohne Verzögerung erfolgen [1, 3], die bei Keimnachweis resistogrammgerecht angepasst werden sollte [8]. Eine Antikoagulation mit intravenös verabreichtem Heparin wird bei fehlender Kontraindikation im Akutstadium empfohlen [3], wobei dies nicht durch randomisierte prospektive Studien belegt ist [1]. Handelt es sich um ein nichtinfektiöses Geschehen, so kann in der 
Tab. 1 Zusammenfassung einzelner Fallberichte bei Thrombose der V. ophthalmica superior

\begin{tabular}{|c|c|c|c|c|}
\hline Patient/in & Symptome & Befund & Therapie & Verlauf \\
\hline $\begin{array}{l}42 \text { Jahre alt, männlich, VE: Z.n. zwei } \\
\text { vorangegangenen tiefen Venenthrom- } \\
\text { bosen und einer Hemikolektomie bei } \\
\text { Kolonkarzinom [2] }\end{array}$ & $\begin{array}{l}\text { Schmerzen und Sehstörun- } \\
\text { gen auf dem linken Auge } \\
\text { nach dem Aufwachen drei } \\
\text { Tage nach Absetzen der } \\
\text { Warfarin-Therapie }\end{array}$ & $\begin{array}{l}\text { Visus: LA:Nnulla Lux } \\
\text { IOD: } 55 \mathrm{mmHg} \\
\text { LA: relatives afferentes } \\
\text { Pupillendefizit, diffuse } \\
\text { hämorrhagische Chemo- } \\
\text { sis, } 3 \mathrm{~mm} \text { Proptosis links } \\
\text { und Ophthalmoplegie }\end{array}$ & $\begin{array}{l}\text { Notfallmäßige Kan- } \\
\text { thotomie mit Kantho- } \\
\text { lyse links } \\
\text { Systemisch: Acetazol- } \\
\text { amid oral, Heparin } \\
\text { i.v., Methylpredniso- } \\
\text { lon } 250 \text { mg i.v. alle } \\
6 \text { Stunden } \\
\text { Topisch: Timolol 0,5\%, } \\
\text { Latanoprost, Dorzol- } \\
\text { amid }\end{array}$ & $\begin{array}{l}\text { Postop. IOD-Senkung } \\
\text { auf } 25 \mathrm{mmHg} \\
\text { Ein Jahr später: Nnulla } \\
\text { Lux, Optikusatrophie } \\
\text { (Rückbildung der } \\
\text { Proptosis und Oph- } \\
\text { thalmoplegie) }\end{array}$ \\
\hline $\begin{array}{l}58 \text { Jahre alt, weiblich, VE: Sichelzellanä- } \\
\text { mie, arterielle Hypertonie [2] }\end{array}$ & $\begin{array}{l}\text { Schmerzen in der rechten } \\
\text { Augenhöhle und Diplopie } \\
\text { nach dem Aufwachen }\end{array}$ & $\begin{array}{l}\text { Visus: OD 20/25 } \\
\text { IOD: normwertig } \\
4 \mathrm{~mm} \text { Proptosis rechts } \\
\text { mit leichten Supradukti- } \\
\text { ons- und Adduktionsde- } \\
\text { fiziten }\end{array}$ & $\begin{array}{l}\text { Orale Warfarin-Be- } \\
\text { handlung }\end{array}$ & $\begin{array}{l}\text { Zwei Wochen später: } \\
\text { Visus rechts 20/20 bei } \\
\text { voller Beweglichkeit } \\
\text { und Auflösung der } \\
\text { Proptosis }\end{array}$ \\
\hline $\begin{array}{l}77 \text { Jahre alt, männlich, VE: HHT, Diabe- } \\
\text { tes mellitus, Bluthochdruck, Hypercho- } \\
\text { lesterinämie, Nikotinabusus [2] }\end{array}$ & $\begin{array}{l}\text { Schmerzhaftes rechtes } \\
\text { Auge, verschwommene } \\
\text { Sicht und Diplopie nach } \\
\text { dem Aufwachen }\end{array}$ & $\begin{array}{l}\text { Visus OD: } 20 / 25 \\
\text { IOD: normwertig } \\
\text { Pupillenreaktion: un- } \\
\text { auffällig. Die Exophthal- } \\
\text { mometrie ergab eine } \\
\text { Proptosis von } 3 \mathrm{~mm} \\
\text { rechts. Leicht einge- } \\
\text { schränkte Beweglichkeit } \\
\text { in allen Positionen }\end{array}$ & $\begin{array}{l}\text { Keine } \\
\text { (eine Antikoagulati- } \\
\text { on wurde aufgrund } \\
\text { des erhöhten Risikos } \\
\text { von systemischen } \\
\text { Blutungen im Zusam- } \\
\text { menhang mit der HHT } \\
\text { nicht eingeleitet) }\end{array}$ & $\begin{array}{l}\text { Proptosis und Diplopie } \\
\text { bildeten sich inner- } \\
\text { halb von } 5 \text { Wochen } \\
\text { spontan zurück }\end{array}$ \\
\hline $\begin{array}{l}23 \text { Jahre alt, männlich (Transgender), } \\
\text { Vorgeschichte: intramuskuläre Testoste- } \\
\text { roninjektionen ( } 50 \mathrm{mg} / \mathrm{ml} \text { wöchentlich } \\
\text { in den letzten } 8 \text { Monaten) im Rahmen } \\
\text { einer geschlechtsangleichenden Hor- } \\
\text { montherapie. VE: SLE, supraventrikuläre } \\
\text { Tachykardie, bilaterale avaskuläre Ne- } \\
\text { krose der Hüfte und Knie, Hypothyreo- } \\
\text { se, Asthma bronchiale [12] }\end{array}$ & $\begin{array}{l}\text { Schmerzhaftes Auge und } \\
\text { verschwommene Sicht } \\
\text { rechts seit zwei Tagen } \\
\text { (unter intramuskulären } \\
\text { Testosteroninjektionen, } \\
\text { Mycophenolat-Mofetil } \\
\text { bei SLE und Hochdosis- } \\
\text { Prednisolon-Einnahme } \\
\text { aufgrund eines Hautaus- } \\
\text { schlags drei Wochen zuvor) }\end{array}$ & $\begin{array}{l}\text { Visus OD: } 20 / 30 \\
\text { IOD: normwertig } \\
\text { Pupillenreaktion: unauf- } \\
\text { fällig } \\
\text { Orthotropie im alter- } \\
\text { nierenden Cover-Test. } \\
\text { Die Exophthalmome- } \\
\text { trie ergab eine Prop- } \\
\text { tosis von } 2 \text { mm rechts. } \\
\text { Fundoskopisch keine } \\
\text { Auffälligkeiten }\end{array}$ & $\begin{array}{l}\text { Heparinperfusor. Ab- } \\
\text { setzen der intramus- } \\
\text { kulären Testosteronin- } \\
\text { jektionen. Der Patient } \\
\text { wurde auf Enoxaparin } \\
\text { und dann auf Warfarin } \\
\text { umgestellt }\end{array}$ & $\begin{array}{l}\text { Verbesserung der Be- } \\
\text { schwerdesymptomatik } \\
\text { unter der Antikoagula- } \\
\text { tion } \\
\text { Versterben } 1,5 \text { Mo- } \\
\text { nate nach Entlassung } \\
\text { aufgrund eines Herz- } \\
\text { stillstandes unklarer } \\
\text { Genese (keine Au- } \\
\text { topsie durchgeführt) }\end{array}$ \\
\hline \multicolumn{5}{|c|}{$\begin{array}{l}\text { Ein Überblick über die aktuelle Literatur zur Thrombose der V. ophthalmica superior zeigt, dass das führende Symptom der plötzliche Exophthalmus nach } \\
\text { dem Aufwachen ist. Die Therapie erfolgt mittels Antikoagulation. Die Visusprognose korreliert mit dem initialen Visus nach Auftreten der Symptome } \\
\text { VE Vorerkrankungen, OD Oculus dexter, IOD intraokularer Druck, HHT hereditäre hämorrhagische Teleangiektasie, SLE systemischer Lupus erythematodes, } \\
\text { Z. n. Zustand nach }\end{array}$} \\
\hline
\end{tabular}

Akutphase eine probatorische Hochdosiskortikosteroidgabe mit ausschleichender Dosierung über mehrere Wochen erfolgen [3]. Führt die Thrombose zur Verdrängung orbitaler Strukturen, beispielsweise mit Affektion des N. opticus, so kann eine Orbitadekompression erforderlich werden [3].

Prognose. Insgesamt weist die inzidentelle Thrombose der V. ophthalmica superior eine günstige Prognose auf, ist in der Regel selbstbegrenzend und lässt sich mit Antikoagulation gut behandeln [8]. Gleiches gilt für posttraumatische und postoperative Thrombosen [8]. Septisch bedingte Thrombosen, die im Zusammenhang mit einer Nasennebenhöhlenentzündung, orbitaler Zellulitis oder nach einer Nasennebenhöhlenoperation auftreten, zeigen häufig einen aggressiven Verlauf mit hohem Komplikationsrisiko [8].

\section{Fazit für die Praxis}

- Zur Abgrenzung anderer Entitäten wie beispielsweise einer arteriovenösen Malformation kann eine orbitale Duplexsonographie oder eine digitale Subtraktionsangiographie erfolgen.

- Eine Spontanthrombose der V. ophthalmica ist selten und sollte bei plötzlichem einseitigem Auftreten in Betracht gezogen werden.

- Relevante systemische Begleiterkrankungen sollten ausgeschlossen und eine ge- naue Anamnese sollte erhoben werden, da eine Unterscheidung in septische und aseptische Genese entscheidend für das weitere Procedere ist.

- Ein universelles Behandlungsprotokoll liegt bislang nicht vor. Bei inzidentellem Auftreten ist eine antikoagulative Therapie wirksam und die Prognose günstig. 


\section{Korrespondenzadresse}

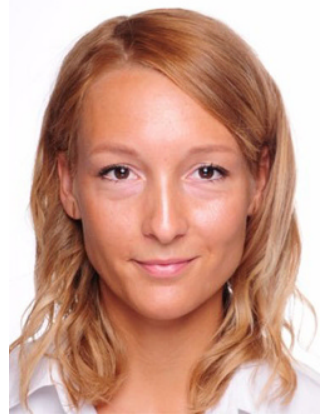

Dr. med. K. Borgardts

Universitätsklinikum Düsseldorf

Moorenstr. 5, 40225 Düsseldorf, Deutschland

KlaraCharlotte.Borgardts@

med.uni-duesseldorf.de

Funding. Open Access funding enabled and organized by Projekt DEAL.

\section{Einhaltung ethischer Richtlinien}

Interessenkonflikt. K. Borgardts, K. Spaniol, C. Trigaux, W. Dahma, M. Kaschner, M. Borrelli und G. Geerling geben an, dass kein Interessenkonflikt besteht.

Für diesen Beitrag wurden von den Autoren keine Studien an Menschen oder Tieren durchgeführt. Für die aufgeführten Studien gelten die jeweils dort angegebenen ethischen Richtlinien. Für Bildmaterial oder anderweitige Angaben innerhalb des Manuskripts, über die Patienten zu identifizieren sind, liegt von ihnen und/oder ihren gesetzlichen Vertretern eine schriftliche Einwilligung vor.

Open Access. Dieser Artikel wird unter der Creative Commons Namensnennung 4.0 International Lizenz veröffentlicht, welche die Nutzung, Vervielfältigung Bearbeitung, Verbreitung und Wiedergabe in jeglichem Medium und Format erlaubt, sofern Sie den/die ursprünglichen Autor(en) und die Quelle ordnungsgemäß nennen, einen Link zur Creative Commons Lizenz beifügen und angeben, ob Änderungen vorgenommen wurden.

Die in diesem Artikel enthaltenen Bilder und sonstiges Drittmaterial unterliegen ebenfalls der genannten Creative Commons Lizenz, sofern sich aus der Abbildungslegende nichts anderes ergibt. Sofern das betreffende Material nicht unter der genannten Creative Commons Lizenz steht und die betreffende Handlung nicht nach gesetzlichen Vorschriften erlaubt ist, ist für die oben aufgeführten Weiterverwendungen des $\mathrm{Ma}$ terials die Einwilligung des jeweiligen Rechteinhabers einzuholen.

Weitere Details zur Lizenz entnehmen Sie bitte der Lizenzinformation auf http://creativecommons.org/ licenses/by/4.0/deed.de.

\section{Literatur}

1. Lai PF, Cusimano MD (1996) The spectrum of cavernous sinus and orbital venous thrombosis: a case and a review. Skull Base Surg 6(1):53-59

2. Lim LH et al (2014) Spontaneous superior ophthalmic vein thrombosis: a rare entity with potentially devastating consequences. Eye 28(3):348-351

3. Kumar J et al (2015) Diagnosis and management of superior ophthalmic vein thrombosis. EyeNet 19(19):35-36

4. Foroozan R et al (2000) Congenital orbital varices causing extreme neonatal proptosis. Am J Ophthalmol 129(5):693-694

5. Rosenblum P, Zilkha A (1978) Sudden visual loss secondary to an orbital varix. Surv Ophthalmol 23(1):49-56

6. Heran F et al (2014) Tumor pathology of the orbit. Diagn Interv Imaging 95(10):933-944

7. Shields JA et al (1984) Demonstration of orbital varix with computed tomography and valsalva maneuver. Am JOphthalmol 97(1):108-110

8. Sotoudeh $\mathrm{H}$ et al (2019) Superior ophthalmic vein thrombosis: What radiologist and clinician must know? Eur J Radiol Open 6:258-264

9. http://www.issva.org/classification. Zugegriffen: 24. Okt. 2021

10. Benoiton LA et al (2017) Management of Orbital and Periorbital Venous Malformation. Front Surg 4:27

11. Smoker WR et al (2008) Vascular lesions of the orbit: more than meets the eye. Radiographics 28(1):185-204 (quiz 325)

12. Park J, Armstrong GW, Cestari DM (2019) Spontaneous superior ophthalmic vein thrombosis in a Transgender man with systemic lupus Erythematosus. LGBT Health 6(4):202-204

\section{U. Pleyer (Hrsg.) \\ Entzündliche Augenerkran- kungen}

Springer 2021, 2. Auflage, 905 S., (ISBN: 978-3-662-60398-7), Hardcover 179,99 EUR

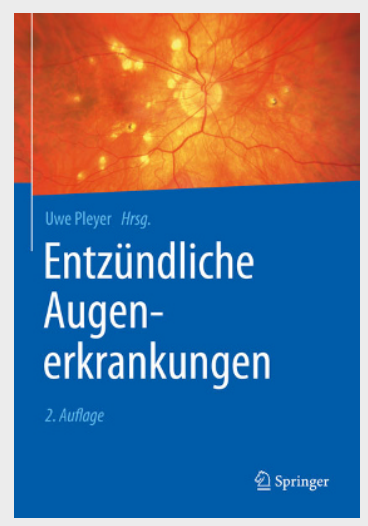

Das Buch vermittelt, mit Herrn Professor Pleyer an der Spitze, umfangreiches Expertenwissen über Pathophysiologie, Diagnostik, Differentialdiagnostik und Therapie entzündlicher Augenerkrankungen.

Es ist auf die praktischen Bedürfnisse der in Praxis oder Klinik tätigen Ophthalmologen ausgerichtet und behandelt eine Vielzahl an entzündlichen Augenerkrankungen, die infektiös oder nichtinfektiös, akut oder chronisch sein können.

Kompakt und übersichtlich werden pathophysiologische Grundlagen, Leitfäden zur Diagnostik und aktuelle Therapieempfehlungen erläutert. Zahlreiche Flussdiagramme, Praxistipps und farbige Abbildungen machen dieses Buch zu einem diagnostischen und therapeutischen Wegweiser durch das Spektrum der entzündlichen Augenerkrankungen.

Für alle Ophthalmologen, aber auch interessierte Internisten, Pädiater und Dermatologen. 